\title{
Corela
}

Cognition, représentation, langage

HS-7 | 2010

Espace, Préposition, Cognition

\section{Quand la préposition dans contribue à l'expression d'une relation logico-temporelle de consécutivité}

Andrée Borillo

\section{OpenEdition}

\section{Journals}

Édition électronique

URL : http://journals.openedition.org/corela/942

DOI : $10.4000 /$ corela.942

ISSN : $1638-573 \mathrm{X}$

Éditeur

Cercle linguistique du Centre et de I'Ouest - CerLICO

Référence électronique

Andrée Borillo, «Quand la préposition dans contribue à l'expression d'une relation logico-temporelle de consécutivité », Corela [En ligne], HS-7 | 2010, mis en ligne le 31 mai 2010, consulté le 01 mai 2019. URL : http://journals.openedition.org/corela/942 ; DOI : 10.4000/corela.942

Ce document a été généré automatiquement le 1 mai 2019.

\section{(c) (i) (3) (2)}

Corela - cognition, représentation, langage est mis à disposition selon les termes de la licence Creative Commons Attribution - Pas d'Utilisation Commerciale - Partage dans les Mêmes Conditions 4.0 International. 


\title{
Quand la préposition dans contribue à l'expression d'une relation logico- temporelle de consécutivité
}

\author{
Andrée Borillo
}

\section{Introduction}

1 De nombreuses études ont montré la possibilité pour la préposition dans de fonctionner hors du domaine spatial. Non seulement pour la constitution de SP de valeur temporelle (cf. Berthonneau 1998, Vandeloise 1999), mais également pour l'expression de divers types de relations abstraites qui mettent en rapport des entités matérielles ou des concepts avec des événements ou des états (cf. Jaeggi 1956, Cadiot 1997).

2 Dans ce cas, il peut s'agir d'un SP fonctionnant dans le cadre de la phrase comme argument d'un verbe ou d'un nom. En tant que complément, il entretient une relation de type statique ou dynamique avec le sujet, ex. il vit dans la peur, il se lance dans des explications, il sombre dans la misère, son engagement dans la lutte, etc. :

(1) Un groupe de femmes et d' hommes abîmés dans la douleur

3 mais il peut avoir ce même type de relation avec un autre complément du verbe, ex. plonger quelqu'un dans la misère, semer le doute dans les esprits, laisser quelqu'un dans l'embarras:

(2) H. défendait la théorie de l'évolution, ce qui l'entraîna dans un conflit avec

l'église catholique.

4 Toujours dans le cadre de la phrase, il peut s'agir également d'un complément circonstanciel renvoyant à des actions, des processus ou des états : dans la défaite, dans la lutte, dans le désordre, dans le calme, dans la confusion, dans le repos, etc. :

(3) Une détonation isolée retentit, sèche et distincte dans le vacarme confus des combats

(4) La nouvelle loi a été adoptée dans la confusion la plus totale 
le reste de la phrase, ce qui, pour (4), pourrait se traduit par des paraphrases du type "La nouvelle loi a été adoptée au beau milieu de la confusion la plus totale" ou "La nouvelle loi a été adoptée alors que régnait la confusion la plus totale".

6 Cependant, concernant plus précisément la catégorie des noms abstraits dénotant des états physiques, mentaux ou psychologiques que l'on rattache aux sensations, aux émotions, aux sentiments, etc., le problème de la fonction d'un SP introduit par dans se révèle plus délicat. En effet, bon nombre de ces noms, qui ont par ailleurs la propriété d'être prédiqués avec des verbes-supports tels que avoir, éprouver, ressentir ou manifester, montrer, afficher (cf. Gross 1995, Negroni-Peyre 1978, Balibar-Mrabti 1995) ont la possibilité d'être régis par la préposition dans, soit que le nom s'accompagne d'un déterminant défini, ex. dans l'affolement, dans la souffrance, dans le désespoir, dans la joie, dans l'enthousiasme, dans la panique, ou d'un possessif, ex. dans son désarroi, dans sa joie, dans son émerveillement, dans sa volonté de, dans son désir de, etc. :

(5)Tous ces honnêtes gens expirent loin des leurs, dans la fièvre, dans la misère,

dans l'horreur, dans le désespoir.

L'étude que je propose ici traite d'un emploi un peu particulier de dans : le syntagme prépositionnel qu'il forme avec des noms abstraits relevant de cette catégorie, ne correspond pas à un complément endophrastique (cf. Guimier $1996:$ 5-6), qu'il soit argumental ou circonstanciel, mais à un complément de type exophrastique, généralement détaché en tête de phrase (cf. Combettes 1998), et qui fonctionne comme un prédicat non-fini (on dit aussi "prédicat second") dans la dépendance de la phrase à laquelle il est rattaché - que j'appellerai ici "phrase d'accueil" (Pacc). Je donne ci-dessous plusieurs exemples de cette construction :

(6) Dans sa hâte, il ouvrit impétueusement la porte sans frapper.

(7) Dans la crainte de la gêner, je détournais d'elle mon regard

(8) Dans son désir de plaire à B., il poussa la complaisance jusqu'à la complicité

(9) Dans le trouble où il était, il heurta du pied contre le seuil de la porte et tomba à

la renverse

8 La relation que la préposition dans établit entre le SP détaché et sa phrase d'accueil Pacc, se comprend dans ces phrases comme une relation temporelle de recouvrement (l'événement dans la phrase a lieu alors que l'état dans le SP se manifeste et perdure), mais aussi comme participant à une relation logique de cause-conséquence ou plutôt de cause-consécutivité, telle qu'elle est définie dans Hybertie 1996 :25, à savoir, une relation logique de consécution factuelle qui s'établit entre deux événements ou entre un état et un événement, l'occurrence de l'un étant motivée par la manifestation ou par l'occurrence de l'autre.

$9 \quad 1$ s'agit ici d'une relation de cause-consécutivité entre un état (s) dans SP et un événement (e) dans Pacc - s étant considéré comme préalable à e et comme motivant son occurrence. La relation est donc à la fois de nature temporelle et logique : l'état (s) exprimé sous forme nominale dans le prédicat second, précède et en quelque sorte motive l'événement (e) contenu dans la phrase d'accueil - ce qui peut être représenté par $\mathrm{SP}(\mathrm{s})=>$ Pacc $(\mathrm{e})$.

On comprend alors que la préposition dans puisse prendre un sens proche d'une préposition de sens causal du type du fait de, en raison de, étant donné, etc., comme on peut le voir ici avec les énoncés (6)-(9): "Du fait de sa hâte" ...."Étant donné son désir de plaire,..." 
11 Cependant, la situation n'est pas aussi claire qu'il pourrait y paraître. D'une part, avec ce même type de SP [dans SN] détaché, la relation logique entre les deux termes peut ne pas être comprise comme un enchaînement de type consécutif :

(10) Dans sa frayeur, elle avait eu la présence d'esprit d'arracher les rideaux en flammes

Dans (10), on ne peut pas donner à "dans sa frayeur" le sens de "en raison de sa frayeur", car la relation logique qui lie les deux prédicats s'oriente au contraire vers un sens d'"opposition concessive", qui pourrait se traduire par les prépositions en dépit de, malgré, etc. Ici, en (10), ce sens oppositif pourrait être explicité à travers l'adverbe concessif cependant ou néanmoins inséré dans la phrase d'accueil :

(10') Dans sa frayeur, elle avait eu cependant la présence d'esprit d'arracher les rideaux en flammes

D'autre part, il ne semble pas qu'on puisse toujours établir une relation logique - qu'elle soit de consécutivité ou d'opposition concessive - entre le SP et la phrase qui suit. Autrement dit, même détaché, un SP [dans SN] n'a pas toujours un statut de prédicat second (je reviendrai sur ce point au $\S 4$ infra) :

(11) Dans la tranquillité de ce jardin, dans le silence tiède de ces montagnes, un grand bruit venu d'en bas nous fait tressaillir

14 Avant d'entamer l'étude de cette construction P[SP[dans SN], Pacc] et d'examiner les diverses conditions qui font que la préposition dans matérialise la notion d'espace temporel (espace non borné puisque le nom dans le prédicat second est de type abstrait, donc d'une extension non mesurable) et en même temps introduit le premier terme d'une relation de cause-conséquence, je voudrais très rapidement faire le rapprochement entre cette fonction de prédicat second du SP détaché et d'autres constructions dans lesquelles on peut avoir également, sous des formes assez comparables, l'expression d'une relation logico-temporelle de même type.

15 Tout d'abord, on peut noter que le SP [dans SN] détaché joue par rapport à la phrase d'accueil le rôle que pourrait remplir un participe présent détaché. On pourrait par exemple forger pour les phrases attestées (7) ou (8), des équivalents approximatifs sur la base d'un participe présent :

(7') Craignant de la gêner, je détournais d'elle mon regard

(8') Désirant plaire à B., il poussa la complaisance jusqu' à la complicité.

De même, on pourrait faire appel à un participe passé traduisant un état résultatif. Sans véritablement constituer une paraphrase, les exemples (12) et (12') pourraient rendre compte d'un effet consécutif de type comparable :

(12) Dans sa colère, il prit le verre et lui lança au visage

(12') Pris par la colère, il prit le verre et lui lança au visage

D'un autre côté, il convient d'indiquer que dans n'est pas la seule préposition à pouvoir exprimer ce type de relation cause-consécutivité entre des situations identifiées comme des actions, des événements ou des états. J'ai montré dans d'autres études (cf. Borillo à paraître), comment des prépositions spatiales telles que à, sur, devant, face à, peuvent elles aussi se faire l'expression d'une telle relation. En effet, ces diverses prépositions dont le sens se différencie pourtant assez nettement lorsqu'elles expriment des relations spatiales entre entités matérielles concrètes, ont la propriété commune de pouvoir constituer un SP détaché remplissant la fonction de prédicat second et entraînant de manière assez comparable une relation de sens consécutif, même si chaque préposition apporte par elle-même des nuances qui lui sont particulières : 
(13) À la réception de cette dépêche, il y eut un effondrement sur tous les marchés

(14) Sur les instructions de S., les miliciens couchèrent les blessés le long d'un petit mur

(15) Sous la pression de l'opinion publique, la Chambre vota la dénationalisation

(16) Devant le refus catégorique de ses interlocuteurs, il donna l'ordre de se retirer

Dans l'étude de dans que je présente ici, je voudrais montrer que la relation logique SP(s) $\Rightarrow$ Pacc(e) auquel participe la préposition dans, repose sans aucun doute sur la catégorie sémantique du nom qu'elle régit, mais également et surtout, sur la conjonction d'un certain nombre de facteurs, les uns relevant du SP lui-même (comme la nature du déterminant et des modifieurs du nom régi), d'autres se rapportant à la phrase d'accueil, et plus particulièrement à la nature du verbe et à des facteurs aspectuels qui s'y manifestent. J'examinerai donc en premier lieu, au $\$ 1$, quelques propriétés définissant la catégorie syntactico-sémantique du nom à l'intérieur du SP; ensuite, au \$2, quelques spécificités propres au SP lui-même, puis au $\$ 3$, quelques conditions plus particulières qui semblent liées à sa construction dans le SP ; enfin au $\S 4$, j'indiquerai pour la phrase d'accueil, quelques traits aspectuels qui participent à la valeur consécutive de son contenu.

\section{Catégorie syntactico-sémantique des noms dans le SP [dans SN]}

19 Pour cette étude, j'ai constitué un corpus de données de plusieurs centaines de phrases relevées, comme je l'ai dit en note2, dans Frantext et dans Google - qui toutes ont la particularité de contenir un SP [dans SN] détaché, dans lequel un état (s) contribue à un effet consécutif sur un événement (e) contenu dans la phrase d'accueil. Sur la base de ces occurrences, j'ai pu établir une liste de près de 200 noms différents que j'ai tenté de caractériser sur la base de propriétés syntactico-sémantiques qui paraissent tout à fait significatives. Cette recension ne prétend pas être exhaustive, car il serait sans doute nécessaire d'entamer une exploration plus large et plus systématique, portant sur des textes plus nombreux et surtout plus variés. J'ai simplement essayé de recueillir un lot d'exemples assez diversifiés, qui pour la plupart peuvent être tenus comme représentatifs de l'usage courant.

Dans ce corpus, les noms qui apparaissent dans un SP [dans SN] détaché participant à un effet consécutif appartiennent à un large ensemble lexical dont les traits saillants sont assez facilement caractérisables, dans lequel trois catégories, voisines mais somme toute différentes, semblent se dégager, que j'ai identifiées sous les termes de 1) Noms de comportement, 2) Noms de sentiment, 3) Noms d'aptitude et de visée prospective.

\subsection{Noms de comportement.}

Dans le premier sous-ensemble, il s'agit de noms renvoyant à un comportement humain (possiblement animal), à une attitude physique ou à une manière d'être passagère - ce qui exclut les qualités qui, elles, sont généralement considérées comme permanentes. Ces noms, que je regroupe sous le terme général de "Noms de comportement", correspondent à des états physiques et/ou psychologiques se traduisant par des attitudes, des manières de se conduire et de se comporter. L'un de ces noms figure dans l'exemple (6) présenté cidessus : 
(6) Dans sa hâte, il ouvrit impétueusement la porte sans frapper.

Ces Noms de comportement sont très nombreux dans la langue. L'une de leurs caractéristiques syntaxiques bien connue est la possibilité qu'ils ont de se construire (en dehors du verbe avoir) avec un verbe-support marquant le caractère d'"extériorisation" ou éventuellement l'effort de "non-extériorisation" - comme manifester, afficher, montrer, révéler, cacher, trahir, dissimuler, etc :

Abattement, abnégation, affolement, agressivité, consternation, cruauté, effarement, ébahissement, étonnement, empressement, épuisement, frénésie, fébrilité, fougue, hâte, maladresse, mauvaise humeur, précipitation, saisissement, véhémence, violence, zèle, etc.

Dans un SP détaché à effet consécutif, ces noms ne sont pas directement en situation de prédication avec ces verbes d'extériorisation, mais se trouvent modifiés par un déterminant possessif ou par une relative en où contenant un verbe d'état tel que être, se trouver (cf 2.2 infra):

(17) Dans sa véhémence, il asséna un coup sur le poignet qui le tenait

(18) Dans l'agitation où il était, il eut bien de la peine à s'endormir

Le caractère d'état de ces noms peut être indiqué de manière explicite (être dans un l'état de consternation, de fébrilité, d'épuisement, etc). Cependant, dans mes relevés, il n'y a guère d'attestation dans un SP détaché à effet consécutif de ce nom marquant la catégorie. Au contraire, la présence dans le SP de noms tels que état, disposition, condition, etc. va souvent de pair avec une interprétation où il ne semble pas $\mathrm{y}$ avoir de relation de consécution entre [dans SN] et le contenu de la phrase d'accueil qui suit :

(19) Dans l' état de passivité où il était tombé, il offrait prise à tous les maux

C'est pourquoi, la présence d'un nom de catégorie comme état tend à marquer le SP comme ne participant pas à une relation de consécution, alors que inversement celle d'autres noms comme mouvement, accès, élan peut orienter vers une interprétation consécutive (cf. exemple (60) § 3.2). Mais ce point demanderait des développements que je n'ai pas la possibilité d'entamer dans le cadre relativement restreint de cette présentation.

27 Il faut préciser que le fait de pouvoir se construire avec des verbes-supports tels que manifester, témoigner, etc. n'est pas un test qui, à lui seul, permet d'identifier un nom de comportement, car ces verbes s'utilisent également avec un grand nombre de noms identifiés comme "Noms de sentiment" (cf. 1.2 infra). Ceci n'a rien d'étonnant car un état affectif que l'être humain peut éprouver, ressentir à l'intérieur de lui-même est très souvent susceptible de s'extérioriser à travers une attitude, une manière d'être - on peut éprouver de la haine, de la joie, de la colère, etc. mais aussi la manifester, la montrer, etc.

\subsection{Noms de sentiment.}

Le deuxième sous-ensemble est constitué par des noms dénotant des états psychologiques, des émotions, des affects, noms que certains auteurs regroupent assez largement dans une seule grande catégorie "Sentiments'" (cf. Gross 1995, Balibar-Mrabti 1995) mais que d'autres tiennent à répartir dans des sous-catégories différentes telles que "Sentiments", "Affects ", "Émotions", etc. (cf. Ortony et al 1994, Van de Velde 1998). Pour simplifier ici, je parlerai globalement de "Noms de sentiment", car dans cette étude, la difficulté n'est pas tant de fixer de manière stricte les frontières de la catégorie à laquelle appartiennent ces noms, que de comprendre pourquoi, dans un ensemble qui paraît 
somme toute assez homogène, certains figurent assez naturellement dans une construction comportant un effet consécutif tandis que d'autres, pourtant tout aussi représentatifs de la catégorie, semblent avoir plus de difficulté à couvrir cet emploi (cf. \$3 infra).

Les Noms de sentiment, peut-être plus nombreux encore que les Noms de comportement, ont en commun la propriété bien connue de se construire (en plus de avoir) avec un verbe-support tel que éprouver, ressentir, nourrir, etc. et de pouvoir entrer dans certains énoncés sous la forme "un sentiment de $\mathrm{N}$ " : un sentiment de haine/ de plaisir/ de terreur, etc.

Mais en même temps, comme je viens de le dire, beaucoup sont susceptibles de se construire avec des verbes-supports que l'on associe aux Noms de comportement (manifester, montrer, témoigner, etc.), lorsque le sentiment se trouve extériorisé à travers des attitudes et des manières de se comporter. En tout cas, l'emploi avec cette deuxième série de verbes marquant l'"extériorisation" est possible avec tous les Noms de sentiment qui, selon mes relevés, peuvent entrer dans un SP détaché à effet consécutif, comme par exemple : agacement, agressivité, allégresse, angoisse, bienveillance, chagrin, colère, compassion, consternation, dépit, désespoir, détresse, écœurement, effroi, embarras, enthousiasme, étonnement, folie, frayeur, fureur, haine, hargne, honte, impatience, indignation, irritation, joie, mépris, panique, rage, rancune, répulsion, sollicitude, surprise, tristesse, trouble, etc.

Concernant cette catégorie des Noms de sentiment (c'est vrai également, mais dans une moindre mesure, pour les Noms de comportement) il n'est pas si facile de détecter les énoncés où un nom figurant dans un SP [dans SN] détaché, contribue à un effet de relation de consécution. Par exemple, si je me réfère à des listes plus ou moins exhaustives de Noms de sentiment auxquelles il est possible d'avoir accès (voir par exemple Gheerbrandt 1978, Balibar-Mrabti 1995), je peux facilement repérer ceux qui, dans mes relevés, figurent dans SP[dans SN] de type détaché, mais ceci ne garantit pas que le SP détaché participe effectivement à une relation consécutive. Pour une interprétation de ce type, il semble que d'autres facteurs doivent être pris en compte; comme on peut le voir à la lecture des deux exemples suivants :

(20) Dans sa tristesse, elle percevait encore ses appels, mais ils ne la touchaient pas

(21) Dans sa détresse, elle a accepté de payer l'équivalent de 150 euros afin qu'il négocie avec le ravisseur,

Dans (20), le SP "dans sa tristesse" semble ne pas constituer le premier terme d'une relation de consécutivité mais apparaître plutôt comme une construction détachée de type "descriptif", au sens où l'entend Combettes, qui sépare les constructions détachées en deux grandes catégories "celles qui prennent une valeur de subordonnée circonstancielle, celles qui jouent un rôle simplement descriptif" (cf. Combettes 1998:47). On peut, semble-t-il, rapprocher ici "dans sa tristesse" de constructions détachées à base d'adjectif ou de participe : "pleine de tristesse", "accablée de tristesse", "noyée dans sa tristesse". Alors que dans (21), "dans sa détresse" est plutôt compris comme "En raison de la détresse qui s'est emparée d'elle,.."

34 Cette différence n'est pas due à l'impossibilité pour tristesse de figurer dans un SP [dans $\mathrm{SN}$ ] à effet consécutif car on voit par exemple cette possibilité dans un énoncé comme 
(22), que l'on comprend comme "En raison de sa tristesse et de sa colère, elle oublia de faire un dernier cadeau à son frère":

(22) Dans sa tristesse et sa colère, elle oublia de faire un dernier cadeau à son frère

À l'inverse, placé dans un autre contexte, on peut trouver détresse dans un SP détaché qui n'est pas à interpréter comme ayant un effet consécutif, mais plutôt comme une relation temporelle de recouvrement :

(23) Mon père a traversé la foule.... Dans sa détresse, il se sentait isolé, incompris; il ne se souciait pas des gens qui l'entouraient

En revanche, il est assez facile de modifier le sens de la phrase vers une interprétation où se manifeste un effet consécutif, si l'on introduit quelques variations sur la catégorie sémantique du verbe ou sur la valeur aspectuelle du temps verbal (cf. \$3 et \$4 infra). Car s'il est important de prendre en compte la catégorie du nom dans le SP [dans SN], et de pouvoir l'identifier comme Nom de sentiment, le fait que le SP participe à un effet consécutif est dû à tout un ensemble de facteurs, et en particulier à des indices présents dans le deuxième terme de la relation représenté par la phrase d'accueil. L'appartenance du nom à la "bonne" catégorie ne peut constituer qu'une condition nécessaire mais pas suffisante.

\subsection{Noms liés aux fonctions (mentales) d'aptitude et de visée prospective.}

Un troisième sous-ensemble lexical, cette fois relativement réduit, regroupe des noms assez différents de ceux des deux premières catégories - différents par leur nature sémantique mais aussi par leur construction au sein du SP [dans SN], comme on le verra ci-dessous au \$2. En effet, ce sont des noms qui ont en commun la propriété syntaxique de ne pas apparaître seuls, mais complétés par un complément nominal, de SN, ou par une complétive infinitive, à Inf/de Inf. En même temps, sur le plan sémantique, ils se regroupent autour de deux notions sensiblement différentes, "aptitude" et "visée prospective".

38 a) Le premier groupe que j'ai appelé "Noms d'aptitude", renvoie à la notion de capacité, de faculté ou d'aptitude à faire, à penser, à imaginer, etc., y compris lorsque cette faculté est prise dans un sens négatif. Ces noms sont en quelque sorte le pendant de verbes comme pouvoir, être capable de, être apte à :

aptitude, capacité, talent, génie, faculté, propension mais aussi incapacité, inaptitude, impuissance, etc.

(24) Dans son impuissance à contrôler la situation, il démissionna de son poste

b) Le deuxième groupe, "Noms de visée prospective " (ou "d'anticipation") se regroupent autour de l'idée de visée, d'intention consciente ou non consciente, de projection dans le futur, de but à atteindre - que cette disposition soit envisagée de manière positive ou négative :

41 Ambition, ardeur, aspiration, attente, intention, désir, soif, besoin, crainte, refus, effort, espoir, envie, répugnance, répulsion, crainte, appréhension, volonté

(25) Dans son besoin de penser contre son milieu, il avoue son impuissance à s'en détacher

Il est assez facile avec certains de ces noms de remplacer dans par la préposition avec, à condition que l'article défini se substitue au possessif: dans/avec le désir de promouvoir... 
dans/avec l'ambition de... dans/avec l'espoir de. Mais on se rapproche alors avec ces noms de compléments traditionnellement appelés compléments de finalité ou de but, construits avec les prépositions en vue de, dans le but de, afin de, pour, dont le sens s'avère relativement différent. de celui auquel participe ici le SP détaché [dans SN] :

(26) Dans l'espoir de stabiliser la situation, les Etats-Unis ont envoyé de nouveaux renforts militaires..,

Pour ces noms du 3ème groupe, le complément qu'ils introduisent, sous la forme d'un syntagme nominal ou d'une proposition infinitive, représente soit le fait de l'aptitude ou l'inaptitude, soit l'objet de l'intention ou de la visée prospective qu'ils expriment. Ces noms ne semblent pas former un ensemble lexical très étendu en français, et en tout cas, dans l'emploi qu'ils peuvent avoir sous forme de SP [dans SN] détaché participant à un effet consécutif, je n'en ai dénombré qu'une trentaine environ.

Considérant l'ensemble des données textuelles sur lesquelles mon étude s'appuie, l'appartenance du nom à l'une de ces trois catégories apparaît comme une condition préalable pour que le SP [dans SN] détaché soit à même de participer à une relation de cause-consécutivité. Il n'en reste pas moins que d'autres conditions doivent être remplies pour que cette relation soit effectivement à l'œuvre dans une phrase.

\section{Quelques spécificités du SP [dans SN] à effet consécutif}

\subsection{Le SP [dans SN] à effet consécutif, confronté à d'autres compléments détachés}

Le SP [dans SN] à effet consécutif se présente comme un complément détaché en ce qu'il ne participe pas à la construction de son contenu propositionnel mais s'y adjoint en tant que prédicat second. La position canonique de ce SP est d'être placé en tête, suivant en cela l'ordre de précédence qui logiquement s'établit entre la manifestation d'un état, s, et ce qui apparaît comme un événement ou une action qui en découle, e, selon le schéma [SP $(\mathrm{s})=>\operatorname{Pacc}(\mathrm{e})]$.

46 Cependant, il n'est pas impossible que le SP détaché soit placé ailleurs dans la phrase, par exemple en finale ou en incise. C'est surtout vrai s'agissant de noms du 3ème groupe Noms d'aptitude et Noms de visée prospective - qui nécessitent pour leur construction un complément de forme nominale ou infinitive, et qui, de ce fait, sont peut-être plus facilement identifiables en tant que prédicat, quelle que soit leur position dans la phrase :

(26') (26) Les Etats-Unis ont envoyé de nouveaux renforts militaires, dans l'espoir de stabiliser la situation

(27) La Renaissance, dans son besoin de liberté, a abandonné la spiritualité.

Cependant, le fait que le SP [dans SN] prenne, en tant que prédicat second, la forme d'un complément détaché, ne doit pas le faire confondre avec d'autres compléments qui peuvent eux aussi se plier à une règle de détachement et être déplacés en tête de phrase.

a) Le premier type de complément auquel on peut penser est celui dans lequel la préposition dans régit un nom prédicatif dénotant une action ou un procès, et non pas un comportement, un état affectif ou un état mental. Les noms que j'ai pu relever sont évidemment très nombreux, parmi lesquels on peut citer : 

en correspondance avec la question En quelles circonstances ? En quelle occasion ? La préposition dans qui l'introduit exprime une relation de localisation temporelle comme pourraient le faire des expressions telles que dans le cours de, au cours de:

(28) Dans la réponse qu'il adresse aux fameux quatorze points du président Wilson, il prend un tour hautain et ironique qui ne lui était point coutumier

(29) Dans sa fuite, l'adolescent, armé d'un pistolet, a tué trois passants,

Il n'est pas rare que le complément détaché trouve une équivalence dans une subordination circonstancielle (cf. Combettes $98: 46$ ) prenant la forme d'une construction participiale ou d'un gérondif. Ainsi, dans (30) "dans sa chute" pourrait être substitué par un participe présent "en chutant, "en tombant" :

(30) Dans sa chute, l'arbre s' était en partie brisé

Normalement, ce type de complément détaché temporel ne constitue pas le premier terme (i.e. le terme représentant la cause) dans une relation de consécutivité. On peut le voir dans les exemples (28) et (29) ci-dessus, où "dans sa réponse", "dans sa fuite" ne peuvent pas être paraphrasés par "du fait de sa réponse " "en raison de sa fuite ". Mais, évidemment, il n'est pas impossible qu'une relation de cause à effet soit le fruit de l'enchaînement temporel des deux événements - le premier pouvant, par sa nature, être considéré comme constituant la cause du second. C'est le cas de (30), où la relation consécutive que l'on établit entre l'événement "la chute de l'arbre" et le fait qu' "il se soit brisé en partie" s'inscrit dans la nature des choses.

Cependant, une telle relation ne pourrait être établie, uniquement sur la base de cette construction, pour n'importe quel couple d'événements; par ex. dans (31) où est exprimée une relation temporelle de recouvrement:

(31) Dans sa chute, il s'était rattrapé machinalement au bord de la nappe

b) Un deuxième type de complément détaché de forme SP [dans SN] semble plus proche du SP prédicat à effet consécutif qui nous intéresse ici, car dans peut s'y appliquer à des noms appartenant à l'une des trois catégories mentionnées au $\S 1$, et notamment à la catégorie des Noms de sentiment (cf. \$1.2). C'est d'ailleurs ce type de complément que j'ai utilisé plus haut dans mes exemples, de (20) à (23), pour illustrer la difficulté d'interprétation que peuvent créer les Noms de sentiment participant à un SP [dans SN] détaché.

57 En effet, dans ces exemples, on a pu voir qu'un Nom de sentiment comme tristesse ou détresse, présent dans un SP [dans $\mathrm{SN}$ ], peut contribuer à une interprétation de type consécutif ou pas, selon la phrase à laquelle le SP est rattaché. Je rappelle ici le couple d'exemples (21) et (23):

(21) Dans sa détresse, elle a accepté de payer l'équivalent de 150 euros afin qu'il négocie avec le ravisseur,

(23) Mon père a traversé la foule.... Dans sa détresse, il se sentait isolé, incompris; il ne se souciait pas des gens qui l'entouraient

Corela, HS-7 | 2010 
chose vaut pour un assez grand nombre de noms. Si l'on prend par exemple frayeur en (32) et (33) ci-dessous, dans les deux cas il s'agit d'un SP détaché, mais le second (33) s'interprète plus facilement que le premier (32) comme participant à un effet consécutif et pouvant se traduire par "du fait de sa frayeur" :

(32) Dans leur frayeur, ils ne savaient quel parti prendre, et le courage leur manquait,

(33) Dans sa frayeur, il laissa échapper son livre et tous les soldats se mirent à rire.

Comme on le verra plus loin aux \$3. et 4, la différence qui, on le voit, ne tient pas au nom inclus dans le SP, peut être rapportée à des facteurs essentiellement aspectuels relevant de la phrase d'accueil.

\subsection{Propriétés syntaxiques du SP [dans SN] à effet consécutif}

60 Une fois définie la nature du nom - ainsi que j'ai tenté de le faire au §1. - le trait le plus marquant de ce SP est le fait que le SN doit être de quelque manière défini et doit entretenir un lien coréférentiel avec le sujet de la phrase d'accueil. Il peut être défini de trois manières, a) par la présence d'un déterminant possessif auprès du nom, b) par la modification du nom par une relative, c) par la modification du nom par un complément de nom ou une complétive infinitive.

\subsubsection{Présence d'un déterminant possessif.}

61 Dans mes relevés, un très grand nombre de SP sont de forme [dans poss $\mathrm{N}$ ]. Le référent $\mathrm{du}$ possessif est un être humain (voir note8 au \$1.1 supra), dénoté dans la phrase d'accueil par un nom ou un pronom remplissant le plus souvent la fonction sujet :

(34) Dans sa1 colère, il1 prit le verre et lui lança au visage

Ceci ne veut pas dire que l'emploi d'un possessif est réservé à ce type de complément. S'agissant des trois catégories de nom concernées, il est bien connu que le possessif, marqueur de coréférence, entre très naturellement dans la relation de méronymie qui lie un comportement avec celui qui le manifeste, un sentiment avec celui qui l'éprouve, une faculté mentale avec celui qui en est le siège - dans les trois cas, $\mathrm{j}$ 'emploierai le terme de "expérienceur" pour désigner la personne concernée.

De ce fait, il n'est pas étonnant de voir le possessif couramment employé avec un nom appartenant à l'une de ces trois catégories - Noms de comportement, Noms de sentiment, Noms d'aptitude et de visée prospective :

64 - Par exemple, cet emploi apparaît fréquemment dans certaines structures prédicatives comportant un Nom de comportement ou un Nom de sentiment, dans lesquelles le verbesupport exprime "l'extériorisation" (cf. \$1.2 supra) comme manifester, afficher, cacher, montrer, etc. :

Paul cache sa tristesse; il manifeste sa joie; il montre sa surpris; il affiche sa volonté

de nuire; son geste trahit sa nervosité, etc.

65 Alors que les verbes ne marquant pas l'extériorisation, qui s'appliquent à la seule catégorie des Noms de sentiment (cf. § 1.2 ci-dessus), ne s'accompagnent pas d'un possessif mais d'un défini:

Paul éprouve quelque tristesse; il a de la peine; il ressent de l'admiration 
-delà de cet emploi très courant, on trouve le possessif dans d'autres circonstances, par exemple dans certaines constructions prédicatives obtenues à partir d'opérations de restructuration bien spécifiées, ex. envier l'enthousiasme de X / envier X pour son enthousiasme (cf. Guillet et Leclère 1981), mais il n'y a pas lieu d'en parler ici. relevant des trois catégories - Nom de comportement, de sentiment, d'aptitude ou de visée prospective - la modification du nom par un possessif ne semble pas faire problème. On peut avoir un possessif par exemple lorsque le SP entre dans la constitution de formes prédicatives conjuguées ou participiales (cf. Van de Velde 1998) telles que être plongé dans SN, s'enfoncer dans SN, s'enfermer dans SN, être perdu dans SN, être noyé dans SN, être abîmé dans SN :

Il est plongé dans sa tristesse/ son désespoir, il s'enferme/ se réfugie dans sa

douleur, il est perdu dans son délire, il est noyé dans son chagrin/dans ses regrets

Il n'est donc pas surprenant de voir ces noms modifiés par un possessif lorsque le SP [dans $\mathrm{SN}$ ], est identifié comme un complément détaché à effet consécutif. En vérité, c'est la totalité des noms des trois catégories qui, sans distinction aucune, peuvent prendre un déterminant possessif, au point que cette présence du possessif peut être considérée comme caractéristique de cette construction (Pour d'autres constructions, il est difficile de faire état de formes prédicatives [V dans poss $\mathrm{N}]$ qui puissent s'appliquer à l'ensemble des noms de ces trois catégories). On peut noter par exemple que parmi les nombreux verbes qui acceptent comme complément argumental un SP [dans SN] où le nom exprime une action, un procès ou un état, très peu sélectionnent des noms renvoyant à des attitudes, des sentiments, des intentions, etc. :

persister dans son refus, s'obstiner dans sa contradiction, s'enferrer dans ses torts, se perdre dans ses explications, fouiller dans ses souvenirs,

69 Seuls des verbes comme être plongé dans, s'enfoncer dans, s'enfermer dans (voir la liste donnée ci-dessus) sont susceptibles de sélectionner des Noms de sentiment :

S'enfoncer dans son erreur/dans son chagrin; s'abîmer dans ses pensées/ dans son désespoir

\subsubsection{La présence d'une relative modifiant le nom.}

a) "Relative en où". Ce type de relative contient le verbe-support avec lequel le nom est susceptible de se construire. On la trouve surtout avec des noms du 2ème groupe - les Noms de sentiment - qui, je l'ai dit (et voir \$2.1 supra), utilisent abondamment la préposition dans pour la construction de métaphores exprimant le rapport entre l'expérienceur et ses états (Cf. Van de Velde, 1998 : 397). Ceci est illustré de deux manières,

71 - Soit le verbe prend comme argument sujet, le nom renvoyant à l'expérienceur (Nexp), et comme argument complément, le Nom de sentiment (Nsent): Nexp être dans/se trouve/ se mettre dans Nsent :

(35) Dans l' agitation où elle se trouvait, cette pensée glissa sur elle sans luifaire de mal

- Soit au contraire Nexp et Nsent correspondent l'un et l'autre à un complément argumental d'un verbe causatif : X mettre/plonger/ précipiter Nexp dans Nsent :

(36) Dans le désespoir où ce refus nous mit, nous n'écoutâmes que la passion 
73 b) "Relative en que/qui". Dans ce type de relative, l'expérienceur (Nexp) figure respectivement le complément/le sujet du verbe. S'il s'agit d'un Nom de sentiment, le Nexp dans la fonction de sujet peut se construire avec un verbe-support de type avoir, éprouver, ressentir, concevoir - Nexp éprouver Nsent. :

(37) Dans le ressentiment qu'il en éprouvait, il fit punir sur-le-champ cet imposteur

74 Tandis que dans la fonction complément, on trouve le Nexp avec des verbes comme saisir, s'emparer, prendre, animer, gagner, etc,.ex. Nsent saisir/gagner/ animer/ s'emparer de Nexp où le Nom de sentiment, Nsent, remplit la fonction de sujet :

(38) Dans la colère qui l'anime contre P., elle le chasse

(39) Dans la rage qui s'était emparée de lui, il se jura d'intercepter les lettres

Il n'est pas étonnant que mes relevés manifestent une bonne attestation de cette deuxième structure où le Nsent a la fonction sujet, car dans cette configuration, le Nom de sentiment déjà en place dans la dépendance de la préposition dans, joue dans la relative qu'il introduit, un rôle d'agent dont la force dynamique est mise en évidence par le verbe qui l'accompagne, qui est très souvent un verbe de type inchoatif (voir. $\$ 4.2$ infra) ex. saisir, s'emparer, prendre, etc. (Dans la rage qui s'était emparée de lui,.. Dans l'émotion qui l'envahit,... Dans la frayeur qui le saisit, ...).

c) Autres types de relative.

77 - On peut noter la présence d'une forme relative du type "qui ETRE le sien/ la sienne"

(40) Dans l'affolement qui était le sien, elle trébucha et bascula dans le vide

78 Cette forme de relative n'apparaît qu'assez rarement, et de plus, elle n'est pas particulière à la construction SP [dans SN], car loin d'être réservée aux Noms de sentiment ou aux Noms de comportement, on la trouve plus encore avec des Noms de qualité comme par exemple, honnêteté, tact, bravoure, naïveté, délicatesse, intelligence, etc qui ne se construisent pas avec la préposition dans, mais plutôt avec la préposition avec:

Avec la bravoure qui était la sienne, Avec l'honnêteté qui est la sienne, etc.

- On peut retenir aussi une forme de relative, assez proche de la précédente : "qui le/la caractérise", qui, elle aussi, est plus appropriée pour une construction avec des Noms de qualité:

Avec la délicatesse qui la caractérise, Avec l'intelligence qui le caractérise, etc., mais qui semble d'un emploi possible avec certains noms dénotant un comportement, ex. fougue, emballement, véhémence, impétuosité :

Avec la véhémence qui le caractérise, avec la fougue qui la caractérise, etc.

81 On peut en avoir le témoignage avec une phrase - la seule que j'ai rencontrée - où une relative de ce type apparaît précisément dans un SP [dans SN] à effet consécutif :

(41) Dans l'impétuosité qui le caractérise, il avait brisé tous les obstacles

\subsubsection{Présence d'un complément de nom ou d'une complétive infinitive.}

82 Un complément sous forme de nom ou de complétive infinitive se rencontre parfois avec les Noms de comportement et les Noms de sentiment. Là aussi, le nom peut être modifié par un possessif ou par un déterminant défini qui marque la coréférence qu'il entretient avec le sujet de la phrase :

(42) Dans la1 joie de retrouver Athènes, Thésée1 oublie de mettre une voile blanche comme il l'avait promis à son père 
(43) Dans la1 surprise de cette irruption soudaine, à peine quelques-unes1 de ces guerrières 1 purent-elles prendre leurs armes Noms à visée prospective) que cette construction, infinitive ou nominale, semble avoir cours. Comme je l'ai dit au § 1.3, ces noms appellent un complément prédicatif, que ce soit sous la forme d'un syntagme nominal ou d'une proposition infinitive introduite par de ou à : avoir le désir de Inf, avoir la crainte de SN/ Inf, avoir l'espoir de SN/Inf, avoir la volonté de Inf, être dans l'incapacité de, être dans l'impossibilité de, etc.

(44) Dans le désir de rendre son fils capable d'exercer ses fonctions, le père se tuait de lui marteler la cervelle à coups de leçons

(45) Dans l'espoir de mettre fin aux combats, il donne l'ordre de hisser un drapeau blanc

(46) Dans la crainte de nouvelles attaques, ils quittèrent leurs maisons et se cachèrent dans la forêt

Les noms de cette catégorie qui, dans mon corpus figurent dans un SP [dans SN] détaché, sont presque toujours accompagnés de ce type de construction, infinitive ou nominale, mais il peuvent apparaitre seuls, sans complément, simplement modifiés par un déterminant démonstratif assurant un lien anaphorique avec ce qui précède : dans cette crainte, dans cet espoir, dans cette incapacité :

(47) Il comptait bien sur son avancement, à la fin de l'année, et dans cet espoir, il avait repris sa vie laborieuse d'employé modèle.

Bien entendu, la présence d'un complément à la forme nominale ou infinitive n'empêche pas la modification de ces noms par un déterminant possessif coréférent avec le sujet de la phrase d'accueil, comme on peut le voir dans les exemples (24)-(25) donnés ci-dessus au $\$ 1.3$ ou dans (48):

(48) Dans son envie de satisfaire les fantasmes de son ami le roi, il est prêt à se mettre au banc de la société

\subsection{Quelques propriétés relatives au nom dans le SP détaché à effet consécutif}

86 Apparemment, il n'est pas suffisant de caractériser le nom dans le SP [dans SN] détaché par son appartenance à l'une des trois catégories décrites au \$1. Certes, cette condition est nécessaire, car tous les noms que j'ai pu relever, figurant dans un SP de ce type sont à ranger dans l'une de ces trois catégories, mais il ne semble pas que tous les noms du français relevant de l'une de ces trois catégories puissent dans cette sorte de construction participer à une relation cause-consécutivité. Il semble qu'il faille aussi tenir compte de propriétés liées à la sémantique du nom, et notamment, introduire la notion d'intensité et la notion d'aspect.

\subsubsection{La notion d'intensité.}

On peut noter que dans ce type de SP détaché, le nom est parfois modifié par un adjectif qui exprime le haut degré ou la forte intensité, comme grand, profond, vif, intense, immense, extrême, ex. grand désarroi, violent dépit, cruel délire, extrême désir, etc.

(49) Dans mon immense détresse, j'avais oublié jusqu'à votre existence

(50) Dans sa vive impatience, il précipita la conclusion de ses affaires 
en tel adjectif ne s'avère pas nécessaire. Même en son absence, on comprend en général que l'intensité ou le degré quantitatif dont le nom est porteur, tend à se situer relativement haut dans l'échelle de gradation. Par exemple, en (50) ci-dessus, en l'absence de l'adjectif "vive", on tend à attribuer tout de même à "impatience" le trait d'une forte intensité.

(50') Dans son impatience, il précipita la conclusion de ses affaires

La plupart des phrases relevées semblent témoigner de cette valeur quantitative, même quand aucun indice ne le signale explicitement. Par exemple, en (51) ci-dessous, "sa crainte" ne saurait être interprétée comme "sa légère crainte" :

(51) Dans sa crainte de manquer le train, il m'avait empoigné par un bras et m'obligeait presque à courir

Ceci est peut-être moins vrai pour les noms du 3ème groupe, que l'on peut rencontrer avec des adjectifs de forte mais aussi de faible intensité, ex. faible, léger, vague :

(52) Nous scrutons tous une nouvelle fois l'horizon dans l'immense espoir d'apercevoir des dauphins

(53) Elle descend les deux marches du bureau et s'avance sur moi, dans le vague espoir de m'intimider

Mais comme je l'ai dit plus haut dans le $\$ 1.1 .3$ et plus particulièrement en note14, il n'est pas sûr que les noms comportant l'idée de visée, d'intention consciente ou non consciente, ou de projection dans le futur, puissent tous constituer un SP[dans SN] participant à un effet consécutif (en particulier ceux avec lesquels dans peut être remplacé par avec).

En tout cas, pour ce qui est des Noms de comportement et des Noms de sentiment, cet aspect d'intensité peut expliquer que les noms figurant le plus massivement dans cette construction SP [dans SN] détachée à effet consécutif, comportent dans leur sémantisme des traits de haut degré ou d'intensité forte. En effet, on remarquera que les exemples que j'ai présentés jusqu'ici contiennent des noms comme véhémence (17), agitation (18) détresse (21), frayeur (33), désespoir (36), colère (38), rage (39), affolement (40). Alors que sont plus rares des noms dénotant une intensité de faible degré, comme par exemple déplaisir, ennui, indolence, mélancolie, etc. Et quand on rencontre un tel nom, il n'est pas certain que le SP [dans SN] entre dans un schéma consécutif où dans s'interprète comme $\mathrm{du}$ fait de, en raison de. On peut voir à ce propos (54) et (55) où l'interprétation de "dans sa mélancolie" est assez incertaine :

(54) Dans sa mélancolie, M. ne prêta pas attention à ce qui lui aurait paru le roulement d'un tonnerre lointain

(55) Dans sa mélancolie, elle souhaitait parfois passer promptement de vie à trépas

\subsubsection{La notion d'aspect.}

93 Les noms figurant dans un SP [dans SN] à effet consécutif sont pour la plupart utilisés avec un sens prédicatif chargé d'une valeur aspectuelle. S'agissant par exemple des noms $\mathrm{du}$ 1er ou du 2ème groupe (Noms de comportement et Noms de sentiment), ce qu'ils dénotent dans ce cas ne représente pas pour l'expérienceur un état habituel, stable ou "installé", mais suggère plutôt la manifestation d'un état atteignant ou sur le point d'atteindre une forte intensité sous l'effet d'une certaine dynamique.

94 - Une façon de mettre en lumière cet aspect inchoatif et/ou culminatif est de voir quel verbe-support serait le plus approprié si l'on substituait au SP un prédicat à base de 
participe passé ou de participe présent. Au vu de l'ensemble des exemples que j'ai pu relever où le $\mathrm{SP}[$ dans $\mathrm{SN}]$ participe à un effet consécutif, il semble que les verbes suggérés sont le plus souvent des verbes dotés d'une certaine dynamique, même s'il sont au participe passé :

saisi par, emporté par, envahi par, submergé par, pris par, transporté par, enflammé par, mis dans,

95 plutôt que des verbes marquant la plénitude d'un état installé, comme perdu dans, noyé dans, enfermé dans, nageant dans, plongé dans : noyé dans la tristesse, nageant dans le bonheur, plongé dans l'angoisse :

(56) Dans sa fureur (i.e. saisi par la fureur), il fuit dans les montagnes, résolu à se venger

(57) Elle reconnut la voix de Pierre. Dans sa joie (i.e. transportée de joie), elle n'ouvrit pas le portail, mais rentra en courant pour annoncer que Pierre était là

Ce sont d'ailleurs ces mêmes verbes qui apparaissent lorsque le nom est modifié par une relative en qui, susceptible de modifier le nom dans le SP, comme on a pu le voir au $\$ 2.2$ ci-dessus (dans la rage qui s'empare de lui, dans l'émotion qui la saisit.., etc.)

- Mais sans avoir besoin de recourir à un verbe, on constate également que l'aspect inchoatif peut être exprimé dans le SP par des adjectifs exprimant cette valeur, ex. brusque, brutal, soudain :

(58) Dans sa brusque colère, il fit appeler le nouveau grand vizir

ou, dans la phrase à l'extérieur du SP, par des adverbes marquant la soudaineté dans le déroulement des faits, ex. aussitôt, sur le coup, immédiatement, impétueusement, sans tarder, d'emblée :

(6) Dans sa hâte, il ouvrit impétueusement la porte sans frapper.

(59) Aussitôt, dans son impatience de prendre sa revanche, il se fait conduire en poste à Rochefort où se trouvait le navire

Plus encore, l'aspect dynamique trouve son expression dans des termes caractérisant l'état dénoté par le nom, ex. mouvement, élan, accès, et en même temps soulignant son intensité, ou même sa violence, ex. crise, poussée, explosion, éclat, flambée, etc. (Ces formes apparaissent de manière relativement courante) :

(60) Dans une crise de folle rage, il voulut se jeter sur sa toile, pour la crever du poing

\section{Facteurs aspectuels dans la phrase d'accueil}

Au-delà de la présence d'adverbes marquant la soudaineté de la situation (aussitôt, immédiatement, impétueusement, etc.), la phrase d'accueil possède des éléments qui concourent à présenter l'événement qui s'y exprime, consécutif à la manifestation de l'état représenté par le SP détaché, soit sous un aspect global, soit sous l'angle inchoatif (i.e. dans sa phase initiale).

\subsection{Le temps du verbe.}

101 Quand il y a lieu d'interpréter le SP [dans SN] détaché avec un sens d'effet consécutif, le verbe de la phrase d'accueil apparaît le plus souvent à un temps qui rend compte du caractère global ou inchoatif de l'événement ou de l'action : essentiellement au passé simple, et dans une plus faible mesure, au présent narratif ou au passé composé : 
(61) Dans la fièvre où il était, il eut une pensée bizarre.

(62) Dans sa fébrilité, il lais tomber le ballon au sol ses mots résultant : yeux de, etc,

négocie avec le ravisseur,

(64) Dans son enthousiasme il renverse la bière qui se trouvait sur la table à côté.

(65) Dans son exaspération, il se lève et frappe du poing sur la table en martelant

On trouve quelques temps verbaux composés comme le plus-que-parfait, qui s'inscrit en général dans une relation d'antériorité et rend compte d'une action terminée ou d'un état

(66) Dans sa hâte, elle avait brisé sa lampe, mais cela lui était bien égal, elle connaissait le chemin par cœur

(67) Dans sa distraction, elle avait oublié ses souliers sur la route.Je les ramassai, je m'avançai vers elle.

(68) Dans sa surprise, il avait laissé sa main gauche passée autour du rideau soulevé et la lumière forte tombait en plein sur le visage de la jeune fille

Dans les cas plus rares où le verbe est à l'imparfait, celui-ci donne à la phrase un sens itératif ou habituel, tout en présentant l'action ou l'événement dans sa phase inchoative ou dans une visée prospective :

(69) Dans sa fougue, il lui arrivait assez souvent de se blesser, surtout au niveau des

(70) Dans son enthousiasme, il cherchait à convaincre ses amis autour de lui

\subsection{Prédominance de verbes de nature ponctuelle et inchoative}

En effet, le verbe de la phrase d'accueil est mis au temps du passé simple ou du présent pour marquer l'aspect global de l'événement ou de l'action donnée comme consécutive, mais il est lui-même le plus souvent de nature ponctuelle ("achèvement") ou inchoative, ex. s'ouvrir, s'emporter, se lever, s'enfuir, s'évanouir, se ruer, etc. :

(71) Dans sa douleur, elle s'échappa en courant et du haut de la berge se jeta à l'eau.

(72) Dans son désespoir, elle s'en prit au juge et lui jeta son voile à la face

Et si cet aspect inchoatif n'est pas le fait du sémantisme du verbe, il peut être dû à un verbe auxiliaire qui l'accompagne, tel que se mettre à, commencer à, se décider à, se hâter

(73) Dans son délire, il se mit à imaginer qu'elle venait le rejoindre.

\subsection{Caractère peu adéquat des verbes statifs}

De toute manière, il est fortement requis que le verbe dans la phrase d'accueil renvoie à une action, un processus ou un changement d'état. Certains exemples fournis au cours de cette étude peuvent en témoigner, tels (20), (23) ou (32) reproduits ci-dessous, qui 
montrent comment la présence d'un verbe statif à l'imparfait dans la phrase d'accueil rend plus difficile une interprétation d'effet consécutif pour le SP [dans SN] détaché :

(20) Dans sa tristesse, elle percevait encore ses appels, mais ils ne la touchaient pas

(23) Dans sa détresse, il se sentait isolé, incompris; il ne se souciait pas des gens qui l'entouraient

(32) Dans leur frayeur, ils ne savaient quel parti prendre, et le courage leur manquait,

Dans l'interprétation que l'on à tendance à donner de ces énoncés, [dans SN] n'est pas compris comme [du fait de SN], [en raison de SN], mais plutôt dans un sens proche de celui de formes participiales mises en apposition : (20) noyée dans sa tristesse, elle..., (23) plongé dans sa détresse, mon père... (31) Abîmés dans leur frayeur, ils... c'est-à-dire de formes exprimant un état stabilisé, en liaison avec un état de choses lui-même de nature statique; ceci étant renforcé par la présence du verbe statif à l'imparfait dans la phrase d'accueil.

Ceci peut-être parce qu'il est assez difficile d'établir une relation logique de consécutivité entre deux situations statiques, $s 1=>s 2$, même si la première comporte une certaine intensité qui peut avoir un effet sur la seconde. Il est plus facile en tout cas d'établir une relation de consécution entre une situation caractérisée par son intensité et l'éventualité qu'elle est susceptible de déclencher, s1=>e2, surtout si cette éventualité e2 s'exprime à travers un verbe à la fois ponctuel et inchoatif, tel que prendre, ouvrir, lancer, employé au passé simple - comme dans les exemples (6) ou (12) donnés au tout début :

(6)Dans sa hâte, il ouvrit impétueusement la porte sans frapper

(12) Dans sa colère, il prit le verre et lui lança au visage

111 On peut dire la même chose pour certains énoncés où la phrase d'accueil est au présent. Il se peut qu'une différence soit perçue pour [dans SN], entre un sens paraphrable par du fait de ou en raison de $\mathrm{SN}$ ou un sens temporel de recouvrement proche de dans le cours de, tout au long de SN. Dans le premier cas, il s'agit d'un verbe ponctuel (achèvement) ou inchoatif, et le présent est nécessairement un présent narratif, ce qui est illustré par (74). Dans le second cas, illustré par (75), le verbe trouver peut avoir une valeur stative proche de avoir, disposer de :

(74) L'infortunée ne peut supporter cet affront; dans son désespoir, elle se suspend à un cordon, et cherche à s'étrangler

(75) Dans son désespoir, elle ne trouve que sa petite servante pour la soutenir

Mais sur ce point, l'étude demanderait sans doute à être approfondie...

\section{BIBLIOGRAPHIE}

Berthonneau A.-M. (1998) "Espace et Temps : quelle place pour la métaphore ?" Verbum XX, 4 : 353-382.

Combettes B. (1998) Les constructions détachées en français, Paris, Ophrys

Balibar-Mrabti A. (1995) Une étude de la combinatoire des noms de sentiment dans une grammaire locale, Langue Française, 105 : 88-97. 
Boissel P., Devarrieux J; (1993) "Les groupes prépositionnels en dans " dans C.Guimier (ed) 1001 circonstants, Presses Universitaires de Caen :

Borillo A. (2009) "Le détachement comme mode d'expression d'une prédication seconde de sens temporel" in Les linguistiques du détachement, Apotheloz D., Combettes B., Neveu F. Berne : Peter Lang.

Borillo A. (à paraître) Examen de la préposition sur dans l'expression d'une relation logicotemporelle de consécutivité, Caen, Presses Universitaires de Caen

Cadiot P. (1997) Les prépositions abstraites en français, Paris, Armand Colin

Combettes B. (1998) Les constructions détachées en français, Paris, Ophrys

Flaux N. Van de Velde D. (2000) Les noms en français, Paris, Ophrys.

Forsgren M. Jonasson K., Kronning H. (eds) (1998) Prédication, assertion, information, Uppsala, Acta Universitatis Upsaliensis.

Gheerbrant F.(1978) La nominalisation et les verbes de sentiment. Thèse de 3è cycle, Paris, Université Paris7.

Guillet A., Leclère C (1981) « Restructuration du groupe nominal », Langages 63, pp 99-125.

Gross M. (1981)"Les bases empiriques de la notion de prédicat sémantique. Formes syntaxiques et prédicats sémantiques ", Langages $63: 7-52$.

Gross M. (1995) «Une grammaire locales de l'expressions des sentiments », Langue Française 105 :70-87

Guimier C. (1996) Les adverbes du français, Paris, Editions Ophrys

Leeman, D. (1985) « Tentative de caractérisation d'un complément circonstanciel : "dans mon affolement, je lâchai mon panier de cerises " ", LINX n¹2 : 97-146

Leeman D. (1998) Les circonstants en question, Paris, Kimé

Leeman D. (1999) "Dans un juron, il sauta sur ses pistolets". Aspects de la polysémie de la préposition dans". Revue de Sémantique et Pragmatique, $6: 71-88$

Mélis L. (1988) "La prédication seconde : présentation" Travaux de linguistique 17 : 7-12

Neveu F. (1998) "Les constructions appositives frontales et la structure informationnelle de l'énoncé", in M. Forsgren, K. Jonasson, H. Kronning (eds) Prédication, assertion, information, Uppsala, Acta Universitatis Upsaliensis : 367-376

Neveu F. (2003) "Détachement, adjonction, discontinuité, incidence...Présentation" Cahiers de Praxématique $40: 7-9$

Negroni-Peyre D.(1978) « Nominalisations par être en et réflexivation » Linguisticae Investigationes, tomme II-1, 127-163

Ortony A., Clore G. Collins A. (1994) The cognitive structure of emotions, Cambridge, Cambridge Univ. Press

Vandeloise C. (1992) "Les analyses de la préposition dans: faits linguistiques et effets méthodologiques", Lexique 11:15-40

Vandeloise C. (1995) "De la matière à l'espace: la préposition dans", Cahiers de Grammaire 20 : 123-145, Cahiers de Grammaire $20: 123-145$ 
Vandeloise C. (1999) "Quand dans quitte l'espace pour le temps", Revue de Sémantique et Pragmatique, $6: 145-162$

Van de Velde (1998) "Alice noyée dans ses larmes" Verbum, tome XX, 4 : 395-403

Vetters C. (2003) «L'aspect global : un effet secondaire d'un contenu procédural? » Cahiers

Chronos $11: 113-131$

\section{RÉSUMÉS}

Etude de la préposition dans, dans la construction d'un syntagme prépositionnel (SP) détaché qui fonctionne à la manière d'un prédicat non-fini par rapport à la phrase à laquelle il se rattache ("phrase d'accueil"), ex. Dans sa hâte, il ouvrit impétueusement la porte sans frapper. Comparable en cela à d'autres prépositions comme à, sur sous, devant ou face à, la préposition dans contribue à l'expression d'une relation logico-temporelle de consécutivité faible qui s'établit entre un état $\mathrm{s}$ exprimé sous forme nominale dans le SP détaché et l'événement e représenté par la phrase d'accueil. Dans prend alors un sens proche de étant donné, du fait de, etc. Cet effet de relation consécutive faible est possible grâce à la conjonction d'un certain nombre de facteurs parmi lesquels : 1) la nature sémantique du nom à l'intérieur du SP, 2) des traits syntactico-sémantiques de la construction du SP lui-même, 3) des conditions aspectuelles sur le verbe et d'autres constituants dans la phrase d'accueil garantissant la valeur consécutive qui s'attache à l'événement qui y est exprimé.

Description of the preposition dans (in) introducing a PP functioning as a non-finite clause adjunct to the main sentence ("host sentence"), ex In his haste, he impetuously opened the door without knocking. In the same way as other prepositions such as à (to), sur (on), sous (under), devant (before) or face à (in front of), the preposition dans (in) contributes to the expression of a temporal-logical relation of weak consecutivity between a state s expressed by a nominal form in the PP and an event e represented by the host sentence. Dans (in) can then be interpreted as étant donné (given), du fait de (because of). This effect of weak consecutivity is based on the conjunction of a certain number of factors such as 1) the semantic nature of the noun inside the $\mathrm{PP}, 2$ ) some syntactic features of the PP internal structure, 3) some aspectual conditions concerning the verb and other constituents within the host sentence, necessary to legitimate the interpretation of a consecutivity relation that can be established between a state, expressed by the PP, and an incoming event to be found in the host sentence.

\section{INDEX}

Mots-clés : relation de consécutivité, effet consécutif, SP détaché, SP prédicat second, noms de comportement, noms de sentiment, noms d'aptitude et de visée prospective, notion d'intensité, facteurs aspectuels, temps du verbe, caractère global ou inchoatif de l'événement

Keywords : consecutivity relation, consécutive effect, adjunct PP, attitude nouns, emotion nouns, aptitude and prospect nouns, aspectual markers, verbal tense, global or inchoative aspect

\section{AUTEUR}

\section{ANDRÉE BORILLO}

Université Toulouse-Le Mirail, CLLE/ERSS, UMR 5263 